\title{
Barriers and facilitators to the implementation of doctor- nurse substitution strategies in primary care: qualitative evidence synthesis (Protocol)
}

Rashidian A, Shakibazadeh E, Karimi- Shahanjarini A, Glenton C, Noyes J, Lewin S, Colvin C, Laurant $M$

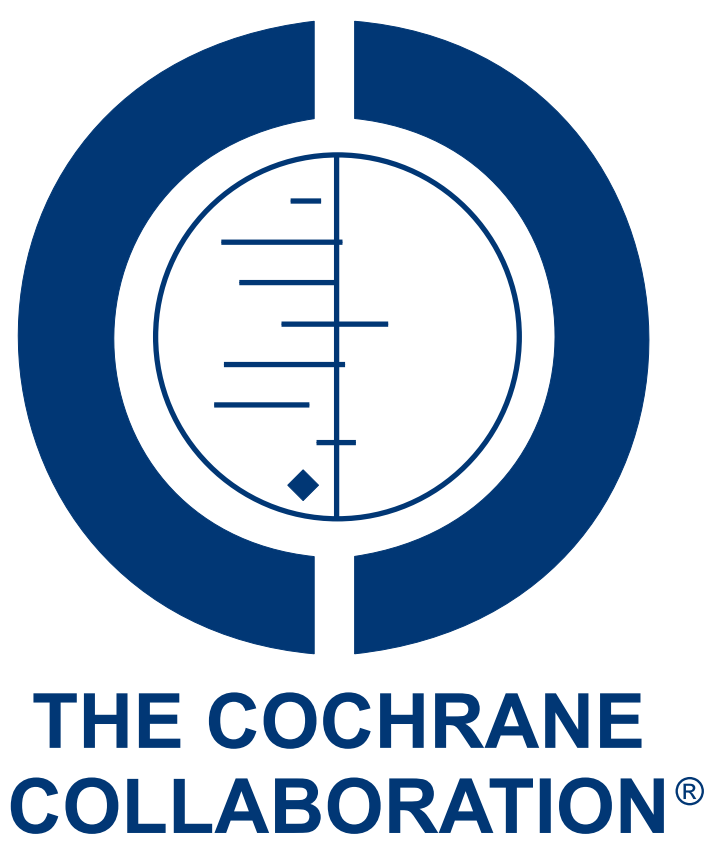

This is a reprint of a Cochrane protocol, prepared and maintained by The Cochrane Collaboration and published in The Cochrane Library 2013, Issue 2

http://www.thecochranelibrary.com

\section{WILEY}

Barriers and facilitators to the implementation of doctor-nurse substitution strategies in primary care: qualitative evidence synthesis (Protocol)

Copyright $\odot 2013$ The Cochrane Collaboration. Published by John Wiley \& Sons, Ltd. 
TABLE OF CONTENTS

HEADER . . . . . . . . . . . . . . . . . . . . . . . . . . . . . . . . . . . . . . . 1

ABSTRACT . . . . . . . . . . . . . . . . . . . . . . . . . . . . . . . . . . . . . . . . . . . . .

BACKGROUND . . . . . . . . . . . . . . . . . . . . . . . . . . . . . . . . . . . . . . . . . . . .

OBJECTIVES . . . . . . . . . . . . . . . . . . . . . . . . . . . . . . . . . . . . . . . . . .

METHODS . . . . . . . . . . . . . . . . . . . . . . . . . . . . . . . . . . . . . . 4

Figure 1. . . . . . . . . . . . . . . . . . . . . . . . . . . . . . . . . . . . . . . . . .

ACKNOWLEDGEMENTS . . . . . . . . . . . . . . . . . . . . . . . . . . . . . . . . . . . . . . . . . . .

REFERENCES . . . . . . . . . . . . . . . . . . . . . . . . . . . . . . . . . . . . . 9

ADDITIONAL TABLES . . . . . . . . . . . . . . . . . . . . . . . . . . . . . . . . . . . . . . . . . 11

CONTRIBUTIONS OF AUTHORS . . . . . . . . . . . . . . . . . . . . . . . . . . . . . . . . . . . 12

DECLARATIONS OF INTEREST . . . . . . . . . . . . . . . . . . . . . . . . . . . . . . . . . . . . . .

SOURCES OF SUPPORT . . . . . . . . . . . . . . . . . . . . . . . . . . . . . . . . . . . . .

Barriers and facilitators to the implementation of doctor-nurse substitution strategies in primary care: qualitative evidence synthesis 


\title{
[Intervention Protocol]
}

\section{Barriers and facilitators to the implementation of doctor- nurse substitution strategies in primary care: qualitative evidence synthesis}

\author{
Arash Rashidian ${ }^{1}$, Elham Shakibazadeh ${ }^{2}$, Akram Karimi- Shahanjarini ${ }^{3}$, Claire Glenton ${ }^{4}$, Jane Noyes ${ }^{5}$, Simon Lewin ${ }^{4}$, Christopher \\ Colvin $^{6}$, Miranda Laurant ${ }^{7}$ \\ ${ }^{1}$ Department of Health Management and Economics, School of Public Health, Tehran University of Medical Sciences, Tehran, Iran. \\ ${ }^{2}$ Department of Midwifery, Zanjan University of Medical Sciences, Zanjan, Iran. ${ }^{3}$ Department of Public Health, Hamedan University of \\ Medical Sciences, Hamedan, Iran. ${ }^{4}$ Global Health Unit, Norwegian Knowledge Centre for the Health Services, Oslo, Norway. ${ }^{5}$ Centre \\ for Health-Related Research, Fron Heulog, University of Wales, Bangor, Bangor, UK. ${ }^{6}$ Centre for Infectious Disease Epidemiology and \\ Research (CIDER), School of Public Health and Family Medicine, University of Cape Town, Cape Town, South Africa. ${ }^{7}$ Scientific \\ Institute for Quality of Health Care, Radboud University Nijmegen Medical Center, Nijmegen, Netherlands \\ Contact address: Arash Rashidian, Department of Health Management and Economics, School of Public Health, Tehran University \\ of Medical Sciences, Poursina Ave, Tehran, 1417613191, Iran. arashidian@tums.ac.ir. arash.rashidian@gmail.com.
}

Editorial group: Cochrane Effective Practice and Organisation of Care Group.

Publication status and date: New, published in Issue 2, 2013.

Citation: Rashidian A, Shakibazadeh E, Karimi- Shahanjarini A, Glenton C, Noyes J, Lewin S, Colvin C, Laurant M. Barriers and facilitators to the implementation of doctor-nurse substitution strategies in primary care: qualitative evidence synthesis. Cochrane Database of Systematic Reviews 2013, Issue 2. Art. No.: CD010412. DOI: 10.1002/14651858.CD010412.

Copyright (C) 2013 The Cochrane Collaboration. Published by John Wiley \& Sons, Ltd.

\begin{abstract}
A B S T R A C T
This is the protocol for a review and there is no abstract. The objectives are as follows:

This review aims to explore factors affecting the implementation of initiatives to substitute doctors with nurses in primary care.

The review has following specific objectives:

- To identify, quality appraise and synthesise qualitative evidence on barriers and facilitators to the implementation of interventions to substitute doctors with nurses in primary care.

- To integrate the findings of this review of qualitative evidence with those of the relevant Cochrane review of effects, so as to enhance and extend understanding of how these complex interventions work and how context impacts on implementation.
\end{abstract}

- To identify hypotheses for sub-group analyses of future updates of the Cochrane systematic review of the effectiveness of substituting doctors with nurses.

Barriers and facilitators to the implementation of doctor-nurse substitution strategies in primary care: qualitative evidence synthesis (Protocol)

Copyright $@ 2013$ The Cochrane Collaboration. Published by John Wiley \& Sons, Ltd. 


\section{B A C K G R O U N D}

\section{Description of the condition}

The majority of countries are facing a chronic shortage and maldistribution of health workers (WHO 2008). This is a key barrier to the achievement of Millennium Development Goals 4, 5 and 6 (WHO 2004). For example, it is acknowledged that human resource shortages in public health care systems play an important role in unsatisfactory health outcomes such as higher maternal mortality rates (Anand 2004; WHO 2008). The problem of human resources shortages is particularly challenging in low- and middle-income countries (LMICs) in sub-Saharan Africa, and in parts of Asia and the Americas. At the same time, the demand for health care is rising. Meeting the Millennium Development Goals of improving maternal and child health, and combating specific diseases (including HIV/AIDS, malaria and tuberculosis (TB)) that are major challenges in LMICs, requires strengthening health systems and equipping them with effective and efficient health service delivery strategies, as well as increasing the coverage and reach of the effective services that are already in place (WHO 2008).

Governments across the world are using a number of approaches to address this problem. One key approach is the moving of tasks from more specialised or highly-trained to less specialised or less highly-trained health workers, for instance by transferring certain tasks from doctors to nurses or midwives. This is sometimes referred to as 'task shifting'; or 'optimising'. By re-organising the health workforce in this way, policy makers hope to make more efficient use of the human resources already available (WHO 2008; WHO 2012). Doctor-nurse substitution may contribute to addressing doctor shortages and reducing physician workload and human resource costs. Anand and Bärnighausen's (Anand 2004) analysis of data from 198 countries suggests that substitution strategies may offer an opportunity for achieving Millennium Development Goals 4, 5 or 6 in LMICs.

\section{Description of the intervention}

Substitution is a process of delegation whereby specific tasks are moved, when feasible, to less highly trained health workers (WHO 2004). The aim of this process is to use more efficiently existing workforce resources in the health sector. This approach can be used to provide a range of health services.

Substitution is not a new strategy. For example, high-income countries such as Australia, the United Kingdom (UK) and the United States of America have extended nurses' roles to include the prescription of routine medications (Cutliffe 2002; Hobson 2010; Stenner 2010). Also, a number of LMICs such as Ethiopia, Haiti, Malawi, Mozambique, Namibia, Rwanda, Uganda and Zambia are currently implementing this strategy to address the chronic shortage of health workers, particularly in the context of generalized HIV epidemics (Assan 2008; Koenig 2004; Morris 2009).

A recent overview of systematic reviews considered the evidence for policy options for human resources, such as substitution or shifting tasks between different types of health workers, and assessed the effectiveness of these strategies in LMICs (Chopra 2008). Results showed that evidence from LMICs is sparse, and the studies are less rigorous than those from high income settings. The authors concluded that more reviews on the effects of policy options to improve human resources in such countries are needed. In addition, a systematic review of substitution (task shifting) strategies for HIV care in Africa noted that the most commonly used intervention was the delegation of tasks from doctors to nurses and other nonphysician clinicians (Callaghan 2010). This review concluded that the delegation of tasks to nurses offered cost-effective care to more patients than a physician-centred model, and others have reached similar conclusions (Colvin 2010; WHO 2008).

\section{How the intervention might work}

The Cochrane Library includes a review exploring the effectiveness of the substitution of general practitioners (family doctors) by nurses in primary care (Laurant 2004a). This review focused on patient outcomes including morbidity, satisfaction with care, quality of life, care processes (e.g., patient compliance, adherence to guidelines), use of resources (including length of consultations, prescriptions, test ordering) and economic variables. The review concluded that nurse-led care was as effective as doctor-led care and associated with higher levels of patient satisfaction and compliance, longer consultations and higher rates of laboratory tests. This review is currently being updated, and its findings are also supported by more recent studies investigating the impact on patient outcomes of nurses or nurse midwives working as substitutes for primary care doctors (Keleher 2009; Sibbald 2004).

By substituting doctors by nurses, nurses take on roles that were previously performed by doctors. The nature of the contribution that nurses substituting for doctors provide in clinical practice is complex and depends on the setting and the roles assigned to nurses, or accepted by them. Different arguments can be put forward to explain why the substitution strategies are employed:

1. Substitution may reduce the cost of providing health care (as nurses are usually paid less than doctors), and hence may be more affordable for the health systems and users of care.

2. Substitution may improve access to care as nurses may provide more coverage; nurses may be available in settings where access to doctors is limited; and doctors may use their free-up time for provision of other services

3. Substitution may even improve quality of care in certain areas, as some clinical tasks, for example patient education, may be better delivered by nurses

4. Substitution may also result in better retention of the nursing workforce via providing new clinical career pathways for 
experienced and educated nurses, further addressing nursing workforce shortages.

These potential relationships between the implementation of substitution strategies and health system objectives, however, are not straightforward and might vary based on the setting and the organisation of care. The complexity of nurse-doctor substitution, its interactions with the contextual factors in each setting as well as the limited effectiveness evidence available, has meant that it is difficult to explain why and how the intervention works, or does not work, in different settings. For example, a randomised trial showed that, at least in the short run, adding nurses to general practice teams did not reduce general practitioners' workload (Laurant 2004b). This trial suggested that nurse practitioners acted as supplements, rather than substitutes, for health care provided by general practitioners. This finding highlights the need to examine how these initiatives are implemented and what factors may explain their effects.

As noted above, one main reason that policy makers may consider substituting doctors with nurses is the expectation that using nurses may reduce costs. Evidence on this is not clear cut (Dierick-van Daele 2009; Hollinghurst 2006; Liu 2012). The Laurant review suggests that longer consultations in nurse-led care may decrease the cost savings of using nurses instead of doctors (Laurant 2004a). However, the exact effect on health service costs was unclear. In addition, Hollinghurst et al. (Hollinghurst 2006) conclude that the costs of employing nurses in primary care are likely to be similar to employing salaried primary care doctors. The way in which the providers (including doctors and nurses) organise their work might also affect the cost-effectiveness of substitution (Liu 2012). In addition, substitution might improve costeffectiveness of care or address equity concerns (for instance, via improving access to those most in need and most likely to benefit from care) without incurring cost savings. Furthermore, the long term cost-effectiveness of a service might differ from short term outcomes, while the former is more difficult to assess.

In 2010, Rashid (Rashid 2010) conducted an integrative qualitative review exploring the benefits and limitations of the recent expansion of clinical roles among nurses working in general practice in the UK. The focus of the study was to establish whether the findings of a previous Cochrane review (Laurant 2004a) were still relevant in the light of recent expansion of nurses' clinical roles in the UK general practice setting. In this study they integrated qualitative evidence with evidence on the effectiveness of nursedoctor substitution in primary care. The study followed a limited approach that only considered qualitative studies conducted in the UK. The author clustered the findings of this review under three themes: the impact on patients, on nurse competence and on $\mathrm{Na}$ tional Health Service policy. According to the findings, patients generally thought that all general practice nurses would be able to deal with simple conditions, but preferred to consult with a general practitioner if they thought it necessary. Indeed, there were concerns about nurses' knowledge base, particularly in diagnos- tics and therapeutics, and their levels of training and competence in roles formerly undertaken by general practitioners. The review concluded that studies in this key area of health care policy are limited.

As most of this limited evidence is from high-income settings, it is not clear to what extent these findings regarding how nurse-doctor substitution works would apply to LMICs.

\section{Why it is important to do this review}

The last few years have seen a strong development in systematic review methodology for integrating and interpreting data from multiple qualitative studies, including within the Cochrane Collaboration (Noyes 2009). The Cochrane Qualitative Research Methods Group has identified around 500 such reviews, although very few of these are of direct relevance to policy makers making health workforce decisions in LMICs. It has been argued that in all countries, including resource poor countries, evidence informed decision making is essential (Chinnock 2005; Garner 1998; Oxman 2010). Policy makers need different types of evidence when choosing appropriate strategies. This includes reliable evidence about local context; but also global research evidence about the effectiveness of different strategies, and about potential barriers and facilitators to their implementation and success.

While the Cochrane intervention review on nurse-doctor substitution concluded that the effectiveness of nurse-doctor substitution initiatives was promising, the results of the included trials were heterogeneous (Laurant 2004a). This finding is not unexpected given the complexity and variability of these types of interventions. In addition, the level of organisation and support used on these trial interventions may have been higher than in real-life settings. If these types of interventions are to be successfully implemented, we need a proper understanding of the factors that may influence their implementation, success and sustainability. Such factors may include the values and preferences of stakeholders and the feasibility and applicability of the intervention for particular settings and health care systems.

There is a growing acknowledgement of the contribution that qualitative research can make to exploring and addressing these questions. As with systematic reviews of effectiveness, reviews of qualitative evidence should be carried out in a systematic and transparent way. By pairing and integrating systematic reviews of effectiveness data with syntheses of qualitative evidence, it will be possible to develop more comprehensive insights and understanding about relevant questions of interest in these topic areas. At least one published Cochrane review has previously prompted a parallel review of qualitative evidence. The Cochrane review of directly observed therapy (DOT) versus self-administered treatment for adherence to TB treatment showed that DOT, despite its widespread use, does not achieve better outcomes than self-administered treatment (Volmink 2007). Two parallel reviews (Munro 2007; Noyes 2007), searched for qualitative studies on factors ex- 
plaining non-adherence to TB treatment so as to shed light on the Cochrane review results. Together, these reviews were not only able to provide compelling evidence regarding the intervention's lack of effect, but also insights that could explain this lack of effect and inform policy and the design of more appropriate interventions (Garner 2007).

Pairing and integrating reviews of effect with reviews of qualitative evidence is equally relevant in the field of health worker interventions, and a body of relevant qualitative research exists. A synthesis of qualitative evidence can help in identifying barriers and facilitators to the success of substitution interventions, including the attitudes and experience of the health workers themselves; but also those of other stakeholders (Harden 2004; Thomas 2008). The previous review on this issue conducted by Rashid 2010 is limited to UK studies only and covered a specific period of time (20042009). The review also does not appear to link qualitative studies with effectiveness studies.

Undertaking this qualitative review is particularly relevant now as the existing Cochrane review on nurse-doctor substitution is currently being updated (Laurant 2004a). The review is also one of a series of reviews of qualitative research that aim to inform the World Health Organization's "Recommendations for Optimizing Health Worker Roles to Improve Access to key Maternal and Newborn Health Interventions through Task Shifting" (OPTIMIZEMNH) (WHO 2012).

\section{O B JECT IVES}

This review aims to explore factors affecting the implementation of initiatives to substitute doctors with nurses in primary care.

The review has following specific objectives:

- To identify, quality appraise and synthesise qualitative evidence on barriers and facilitators to the implementation of interventions to substitute doctors with nurses in primary care.

- To integrate the findings of this review of qualitative evidence with those of the relevant Cochrane review of effects, so as to enhance and extend understanding of how these complex interventions work and how context impacts on implementation.

- To identify hypotheses for sub-group analyses of future updates of the Cochrane systematic review of the effectiveness of substituting doctors with nurses.

\section{METHODS}

\section{Criteria for considering studies for this review}

\section{Types of studies}

This is a systematic review of primary qualitative studies. We will employ a broad definition of qualitative studies and include all studies that utilise qualitative methods for data collection (including focus group and individual interviews, observation and document analysis) and that utilise qualitative methods for data analysis. We will exclude studies that collect data using qualitative methods but do not analyse the data qualitatively.

We will include the following types of studies:

1. Qualitative studies linked to the effectiveness studies included in the Cochrane review of the effectiveness of nursedoctor substitution in primary care (Laurant 2004a) (and the update of this review, which is nearing completion). These include qualitative studies conducted before the effectiveness studies as part of the intervention design process, qualitative process evaluation studies conducted alongside the effectiveness studies, qualitative studies conducted within or specifically funded to run alongside effectiveness studies, and qualitative studies that have followed completion of the effectiveness studies but utilise the same groups of participants.

2. Other qualitative studies that report intervention development, intervention descriptions, implementation and process evaluation to enhance understanding of intervention development, and implementation issues in different contexts.

3. Mixed method studies, provided that it is possible to extract the findings derived from qualitative research.

\section{Types of participants}

The review will include studies that focus on the experiences and attitudes of stakeholders about nurse-doctor substitution. Relevant stakeholders include nurses, patients and their families/carers, the general public, policy makers, programme managers, other health workers, and any others directly involved in or affected by the substitution.

\section{Types of interventions}

We will include studies of the substitution of doctors with nurses and of expanding nurses' roles in community or primary care. For the purposes of this review, we have used the same definition of substitution as in the Cochrane review of effects on this topic, i.e. as "the situation where task(s) formerly performed by one type of professional (i.e. doctor) are transferred to a different type of professional (i.e. nurse), usually with the intention of reducing cost or addressing workforce shortages" (Laurant 2004a).

We have defined primary care as the first level of contact with formal health services, i.e. as those services that "provide first contact and ongoing care for patients with all types of health problems. It includes family practice or general practice, outpatient 
settings, and ambulatory primary care settings (excluding accident and emergency)" (Laurant 2004a). Primary care may be delivered in the community or in a primary care facility (Wiley-Exley 2007; van Ginneken 2011).

\section{Types of outcome measures}

Type of phenomena of interest: The synthesis will include studies where the phenomenon of interest is a description and interpretation of the experiences and attitudes of stakeholders towards the substitution programmes or towards expanding nurses' roles.

\section{Search methods for identification of studies}

\section{Electronic searches}

We will search the following electronic databases for eligible studies:

- CINAHL (EbscoHOST)

- MEDLINE (OvidSP)

- MEDLNE In-Process \& Other Non-Index Citations (OvidSP)

Using guidelines developed by the Cochrane Qualitative Research Methods Group for searching for qualitative evidence (Noyes 2009) as well as a modified version of the search developed for the Laurent effectiveness review on nurse-doctor substitution (Laurant 2004a), we will develop search strategies for each database. We present the strategy for MEDLINE in Table 1. Strategies will be tailored to the other databases and reported in the review.

Previous methodological work has demonstrated that the CINAHL database is the most important resource for qualitative evidence (Flemming 2007). Moreover, Flemming 2007 showed, for a specific review of qualitative evidence, that all of the studies finally included in the review were identified in the CINAHL search. We therefore decided that instead of adding further databases to those listed above, we would follow alternative routes to ensure that relevant studies are identified (see Searching other resources below). A recent integrative (qualitative) systematic review followed a similar approach (Desborough 2012).

Searches will be limited to English for feasibility reasons, given that it is extremely time-consuming and costly to undertake full text translation of qualitative papers into English for inclusion in the review. There will be no date or geographic restrictions.

\section{Searching other resources}

- In addition to our searches of the above-mentioned databases, we will also conduct 'related article' searches in PubMed for all the studies included in the review.

- We will contact experts in the field, and scan reference lists of relevant studies.
- We will search the reference lists of all the included studies and key references (i.e. relevant systematic reviews).

- We will search for any relevant papers that may have cited the included papers and key references (i.e. forwards citation search) in the ISI Web of Science (both the Science Citation Index and Social Science Citation Index).

- We will also conduct individualized searches for qualitative studies that might be linked to, or relevant to, the studies included in the Cochrane nurse-doctor substitution effectiveness review (Laurant 2004a). This will involve: contacting the authors of the effectiveness studies; searching in Pubmed for other articles published by the authors of the effectiveness studies; and conducting 'related article' searches in PubMed for each study included in Laurant 2004a.

\section{Data collection and analysis}

\section{Selection of studies}

We will collate records identified from different sources into one database using reference management software to remove duplicates. Two review authors will then independently assess the titles and abstracts of the identified records to evaluate their potential eligibility and those that are clearly irrelevant to the topic of this review will be discarded at this stage. The full text of all the papers likely to be relevant will be retrieved and assessed independently by two review authors, based on the review's inclusion criteria. At all stages, disagreements between the authors will be resolved via discussion or, if required, by seeking a third review author's view. Where appropriate, we will contact the study authors for further information.

\section{Data extraction and management}

We will perform data extraction using a data extraction form designed specifically for this review and informed by the Supporting the Use of Research Evidence (SURE) framework for identifying factors affecting the implementation of a policy option (SURE Collaboration 2011). This framework includes the following factors: (a) knowledge and skills; attitudes regarding programme acceptability, appropriateness and credibility; and motivation to change or adopt new behaviours among recipients of care, providers of care, and other stakeholders; (b) health system constraints (including accessibility of care, financial resources, human resources, educational system, clinical supervision, internal communication, external communication, allocation of authority, accountability, management and or leadership, Information systems, facilities, patient flow processes, procurement and distribution systems, incentives, bureaucracy, and relationship with norms and standards); and (c) social and political constraints (including ideology, short-term thinking, contracts, legislation or regulations, 
donor policies, influential people, corruption, and political stability).

We will also collect data on the types of nurses (e.g. registered nurses versus nurse practitioners) and the types of substitution strategies in order to develop a map of interventions. These 'typologies' will help to provide a better understanding of factors that contribute to the success or failure in the implementation of substitution.

We will extract additional information concerning the first author's name; year of publication; language; country of study; clinical area; setting of the study (primary health centre or community; rural/urban, etc); and important observations for development and interpretation of the analysis framework.

We will conduct a pilot trial of the data extraction form to check its adequacy, and make changes if necessary. In addition, we will add categories/topics to the list derived from the SURE framework, as data extraction progresses and new areas are identified.

\section{Assessment of risk of bias in included studies}

Appraisal of study quality: Our inclusion criteria specify that included studies need to use both qualitative data collection and analysis methods. This criterion constitutes a basic quality threshold as we will exclude studies that have used qualitative methods to collect data but not to analyse these data. In addition, to assess the methodological quality of included studies, two authors will apply a quality appraisal framework to each study. Appraisal will be performed using an adaptation of the Critical Appraisal Skills Programme (CASP) quality assessment tool for qualitative studies (Critical Appraisal Skills Programme 2010), as in other reviews of qualitative evidence (Carlsen 2007; Munro 2007). This tool includes the following 14 questions:

1. Is this study qualitative research?

2. Are the research questions clearly stated?

3. Have ethical issues been taken into consideration?

4. Is the qualitative approach clearly justified?

5. Is the approach appropriate for the research question?

6 . Is the study context clearly described?
7. Is the role of the researcher clearly described?

8. Is the sampling method clearly described?

9. Is the sampling strategy appropriate for the research question?

10. Is the method of data collection clearly described?

11. Is the data collection method appropriate to the research question?

12. Is the method of analysis clearly described?

13. Is the chosen analytical approach suitable for addressing the research question?

14. Are the claims made supported by sufficient evidence?

We will conduct a pilot trial on three included studies to assess the feasibility of the use of this tool and ensure integrity of the assessment.

Since the aim of the review is to obtain a more comprehensive understanding of the factors that may influence programme success, we will include studies that meet our inclusion criteria regardless of study quality. We will use the quality assessment when judging the relative contribution of each study to the development of explanations and relationships, as described in more detail below. In addition, it has been noted that poor quality studies tend to contribute less to the synthesis. Therefore, the synthesis becomes 'weighted' towards the findings of the studies which have better quality. Also, there is currently no consensus among qualitative researchers on the role of quality criteria and how they should be applied, and there is ongoing debate about how study quality should be assessed for the purposes of systematic reviews (Atkins 2008).

Appraisal of certainty of review findings: Few methods for assessing the certainty of findings drawn from syntheses of qualitative evidence have been developed. To assess how much certainty can be placed in the qualitative evidence for each review finding, we have therefore chosen to apply a novel approach, which we refer to as the CerQual (certainty of the qualitative evidence) approach (Figure 1). By certainty we mean how likely it is that the review finding happened in the contexts of the included studies and could happen elsewhere. 
Figure I. Assessing the certainty of findings from syntheses of qualitative evidence: the CerQual approach

\section{Overall aim: To assess the degree of}

\section{CERTAINTY}

of the qualitative review finding.

(How likely is it that the review finding happened in the study contexts

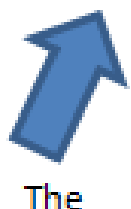

METHODOLOGICAL QUALITY

of the individual studies

that fed into the qualitative

review finding

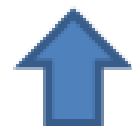

$$
\begin{gathered}
\text { Methodological quality (i.e } \\
\text { how well the studies were } \\
\text { conducted) is assessed by a } \\
\text { methodological appraisal } \\
\text { tool such as CASP }
\end{gathered}
$$
and could happen elsewhere?)

In this approach our assessments of certainty are based on two factors: the 'methodological quality' of the individual studies contributing to a review finding and the 'plausibility' of each review finding.

Findings that are drawn from well-conducted studies can be regarded as more dependable (Lincoln 1985). We will therefore appraise how well the individual studies which contributed to the evidence of a review finding were conducted (methodological quality), using an adaptation of the Critical Appraisal Skills Programme (CASP) quality-assessment tool for qualitative studies (Critical Appraisal Skills Programme 2010).

In addition to appraising the methodological quality of the individual studies that contribute to a review finding, we will also assess the 'plausibility' of each review finding. We will assess plausibility by looking at the extent to which we are able to identify a clear pattern across the individual study data. This pattern could include, for example, circumstances where the review finding is consistent across multiple contexts or where the review finding incorporates explanations for any variations across individual studies. The plau- sibility of the review findings may be further strengthened if the individual studies contributing to the finding are drawn from a wide range of settings.

We will use three levels to indicate the certainty of the qualitative evidence - high, moderate and low. We will rate findings drawn from generally well-conducted studies - and showing high levels of plausibility - to be of 'high' certainty. Findings will be assessed as 'moderate' certainty where there are concerns regarding either the methodological quality of the studies or the plausibility of the review finding. Where there are concerns regarding both the methodological quality of the studies and the plausibility of the review finding, the finding will be assessed as being of 'low' certainty.

The CerQual approach is similar to the GRADE approach (Guyatt 2011) in the sense that both approaches aim to assess the certainty of (or confidence in) the evidence, and both also rate this certainty for each finding across studies. The GRADE approach also bases its judgement of certainty on an assessment of the quality of the evidence and other factors, including consistency across studies. 
However, GRADE is designed to assess the certainty of evidence regarding the effectiveness of an intervention, and is therefore not suitable for use when appraising the certainty of evidence regarding other types of questions and data, including questions about people's perceptions and experiences that are assessed using qualitative methods. CerQual provides an approach that may be used for findings derived from syntheses of qualitative evidence.

The CerQual approach is also similar to one used by Goldsmith et al (Goldsmith 2007). In their synthesis of qualitative research, they assess the overall quality of the evidence for each individual finding by evaluating the quality, consistency and directness of the evidence. We have chosen not to refer to the directness of the evidence as it can be argued that, in the context of qualitative evidence syntheses, this dimension needs to be assessed by the user/s of the evidence.

As a final step, we will prepare summary tables of the findings of the qualitative evidence synthesis. These 'Summary of Qualitative Findings' tables will be similar to the 'Summary of Findings' tables used in Cochrane reviews of effectiveness and will summarise the key findings, the certainty of evidence for each finding, and also provide an explanation of the assessment of the certainty of the qualitative evidence.

The CerQual approach is also being used in another Cochrane Review of qualitative evidence (Glenton 2013).

\section{Assessment of heterogeneity}

We will record differences across the studies with regard to settings, human resources available in the programme and the nature or the tasks being substituted, for example, and these will inform the analyses (see Subgroup analysis and investigation of heterogeneity).

\section{Data synthesis}

We will synthesise qualitative evidence to enhance understanding of questions regarding 'what works for whom and in what context', and to identify 'barriers' and 'facilitators' to the implementation of doctor-nurse substitution strategies. We will conduct a thematic analysis informed by the framework approach used for the analysis of primary qualitative data (Ritchie 1994). The method has been specifically developed for the analysis of qualitative data for policyoriented projects, and will help the review authors focus on the objectives of the review (Lloyd Jones 2005; Thomas 2008). The method has been used successfully by the review authors for policy oriented primary studies in the UK and Iran (Jafarisirizi 2011; Rashidian 2008) and in systematic reviews of qualitative evidence (Noyes 2007).

As in primary qualitative research, we will exploit our theoretical knowledge and the literature to develop a preliminary a priori thematic framework, based on the SURE framework outlined above
(SURE Collaboration 2011). We will adapt this framework using the themes and ideas that emerge through the analysis, while considering other potentially relevant theoretical approaches (Hansen 2010; Chen 2011). The preliminary framework will be discussed in a series of meetings of the core review team. The framework will then be checked against the primary studies through a process of reading and rereading the studies (familiarisation process). Key themes will be updated and refined through this process. All the included studies will be read until no new themes emerge from these studies, and agreements reached on the definition and boundaries of each theme identified. The process of refining the thematic framework will follow an inductive approach, based on the findings of the primary studies.

The text of each primary study will be indexed, by at least review authors, using the codes relating to the themes and sub-themes of the thematic framework. Sections of the studies may be indexed with one or more codes (cross indexing) wherever appropriate. The emerging themes and concepts will be transferred to analysis tables ('charts'), so that the columns and rows of the table reflect the studies and the sub-themes (Miles 1994). The 'chart' will enable us to compare the results obtained in different studies across different themes and sub-themes, and to compare the findings of different studies for each theme. The primary studies will be consulted in the course of the analysis and further extracts may be added to the chart whenever it is necessary.

In the final stage of the analysis, we will juxtapose the key findings from this review of qualitative evidence with those of the Cochrane review of effectiveness (Laurant 2004a) to form integrated matrices of evidence. We will then express the combined quantitative and qualitative synthesis using a narrative summary technique, with the aim of explaining the impacts (or lack of impact) seen in the effectiveness review.

\section{Subgroup analysis and investigation of heterogeneity}

We will conduct sub-group analyses comparing the findings of the studies originating from the LMICs with other studies. This will enable us to generate evidence that is directly relevant to LMICs, develop hypotheses on the applicability of studies from high income countries to LMICs, and draw lessons from high income countries for use in LMICs.

\section{ACKNOWLEDGEMENTS}

We thank Marit Johansen and Andrew Booth for their valuable support in developing the search strategies for this review. We thank Benedicte Carlsen and Heather Munthe-Kaas for their contributions to the development the CerQual approach.

Barriers and facilitators to the implementation of doctor-nurse substitution strategies in primary care: qualitative evidence synthesis 


\section{R E F E R E N C E S}

\section{Additional references}

Anand 2004

Anand S, Barnighausen T. Human resources and health outcomes: cross-country econometric study. Lancet 2004; 364:1603-9.

Assan 2008

Assan A, Mudender F, Brentlinger P, Vallejo J, Bacon O, Martinez P, et al.Task-shifting in Mozambique: evaluating antiretroviral therapy as newly implemented by mid-level health professionals. AIDS 2008 - XVII International AIDS Conference. Abstract MOPE0790. 2008.

Atkins 2008

Atkins S, Lewin S, Smith H, Engel M, Fretheim A, Volmink $\mathrm{J}$. Conducting a meta-ethnography of qualitative literature: lessons learnt. BMC Medical Research Methodology 2008;8 (21). [DOI: 10.1186/1471-2288-8-21]

Callaghan 2010

Callaghan M, Ford N, Schneider H. A systematic review of task-shifting for HIV treatment and care in Africa. Human Resources for Health 2010;8:8.

\section{Carlsen 2007}

Carlsen B, Glenton C, Pop C. Thou shalt versus thou shalt not: a meta-synthesis of GPs' attitudes to clinical practice guidelines. British Journal of General Practice 2007;57(545): 971-8.

Chen 2011

Chen HT, Garbe P. Assessing program outcomes from the bottom-up approach: An innovative perspective to outcome evaluation. New Directions for Evaluation 2011;2011(130): 93-106.

Chinnock 2005

Chinnock P, Siegfried N, Clarke M. Is evidence-based medicine relevant to the developing world?. PLoS Medicine 2005;2(5):e107.

\section{Chopra 2008}

Chopra M, Munro S, Lavis JN, Vist G, Bennett S. Effects of policy options for human resources for health: an analysis of systematic reviews. Lancet 2008;371:629-30.

\section{Colvin 2010}

Colvin CJ, Fairall L, Lewin S, Georgeu D, Zwarenstein M, Bachmann MO, et al.Expanding access to ART in South Africa: the role of nurse initiated treatment. South African Medical Journal 2010;100(4):210-2.

Critical Appraisal Skills Programme 2010 Critical Appraisal Skills Programme (CASP). 10 questions to help you make sense of qualitative research. England: Critical Appraisal Skills Programme 2010. Available from: http://www.casp-uk.net/wp-content/uploads/2011/11/ CASP'Qualitative A Appraisal 'Checklist' 14oct 10.pdf.

Cutliffe 2002

Cutliffe JR. The beguiling effects of nurse-prescribing in mental health nursing: re-examining the debate. Journal of Psychiatric and Mental Health Nursing 2002;9(3):369-75.
Desborough 2012

Desborough J, Forrest L, Parker R. Nurse-led primary healthcare walk-in centres: an integrative literature review. Journal of Advanced Nursing 2012;68(2):248-63.

Dierick-van Daele 2009

Dierick-van Daele ATM, Metsemakers JFM, Derckx EWCC, Spreeuwenberg C, Vrijhoef HJM. Nurse practitioners substituting for general practitioners: randomized controlled trial. Journal of Advanced Nursing 2009;65(2):391-401.

Flemming 2007

Flemming K, Briggs M. Electronic searching to locate qualitative research: evaluation of three strategies. Journal of Advanced Nursing 2007;57(1):95-100.

Garner 1998

Garner P, Kale R, Dickson R, Dans T, Salinas R. Implementing research findings in developing countries. BMJ 1998;317:531-5.

\section{Garner 2007}

Garner P, Smith H, Munro S, Volmink J. Promoting adherence to tuberculosis treatment. Bulletin of the World Health Organization 2007;85(5):404-6.

\section{Glenton 2013}

Glenton C, Colvin C, Carlsen B, Swartz A, Lewin S, Noyes J, Rashidian A. Barriers and facilitators to the implementation of lay health worker programmes to improve access to maternal and child health: qualitative evidence synthesis. Cochrane Database of Systematic Reviews 2013, Issue Forthcoming.

Goldsmith 2007 Goldsmith MR, Bankhead CR, Austoker J. Synthesising quantitative and qualitative research in evidence-based patient information. Journal of Epidemiology and Community Health 2007;61(3):262-70.

Guyatt 2011

Guyatt G, Oxman AD, Akl EA, Kunz R, Vist G, Brozek $\mathrm{J}$, et al.GRADE guidelines: 1. Introduction-GRADE evidence profiles and summary of findings tables. Journal of Clinical Epidemiology 2011;64:383-94.

Hansen 2010

Hansen MB, Vedung E. Theory-based stakeholder evaluation. American Journal of Evaluation 2010;31(3): 295-313.

Harden 2004

Harden A, Garcia J, Oliver S, Rees R, Shepherd J, Brunton G, Oakley A. Applyingsystematic review methods to studies of people's views: an example from public health research. Journal of Epidemiology and Community Health 2004;58(9): 794-800.

Hobson 2010

Hobson RJ, Scott J, Sutton J. Pharmacists and nurses as independent prescribers: exploring the patient's perspective. Family Practice 2010;27(1):110-20. 


\section{Hollinghurst 2006}

Hollinghurst S, Horrocks S, Anderson E, Salisbury C. Comparing the cost of nurse practitioners and GPs in primary care: modelling economic data from randomised trials. British Journal of General Practice 2006;56:530-5.

\section{Jafarisirizi 2011}

Jafarisirizi M, Rashidian A, Abolhasani F, Mohammad K, Yazdani S, Parkerton P, et al. Space or no space for managing public hospitals: a qualitative study of hospital autonomy in Iran. International Journal of Health Planning and Management 2011;26(3):e121-37. [DOI: 10.1002/ hpm.1050]

\section{Keleher 2009}

Keleher H, Parker R, Abdulwadud O, Francis K. Systematic review of the effectiveness of primary care nursing. International Journal of Nursing Practice 2009;15(1):16-24.

\section{Koenig 2004}

Koenig SP, Leandre F, Farmer P. Scaling up HIV treatment programmes in resource-limited settings: the rural Haiti experience. AIDS 2004;18(Supplement 3):S21-5.

\section{Laurant 2004a}

Laurant M, Reeves D, Hermens R, Braspenning J, Grol R, Sibbald B. Substitution of doctors by nurses in primary care. Cochrane Database of Systematic Reviews 2004, Issue 4. [DOI: 10.1002/14651858.CD001271.pub2]

\section{Laurant 2004b}

Laurant MG, Hermens RP, Braspenning JC, Sibbald B, Grol RP. Impact of nurse practitioners on workload of general practitioners: randomised controlled trial. BMJ 2004;328:927. [DOI: http://dx.doi.org/10.1136/ bmj.38041.493519.EE]

\section{Lincoln 1985}

Lincoln YS, Guba EG. Naturalistic enquiry. London: Sage, 1985.

Liu 2012

Liu N, D'Aunno T. The productivity and cost efficiency of models for involving nurse practitioners in primary care: a perspective from queuing analysis. Health Services Research 2012;47(2):594-613.

\section{Lloyd Jones 2005}

Lloyd Jones M. Role development and effective practice in specialist and advanced roles in acute hospital settings: systematic review and meta-synthesis. Journal of Advanced Nursing 2005;49(2):191-209.

Miles 1994

Miles MB, Huberman AM. Qualitative data analysis: an expanded sourcebook. London: Sage, 1994.

\section{Morris 2009}

Morris MB, Chapuli B, Chi B, Mwango A, Chi HF, Mwanza J, et al.Use of task-shifting to rapidly scale-up HIV treatment services: experiences from Lusaka, Zambia. BMC Health Services Research 2009;9:5.

\section{Munro 2007}

Munro SA, Lewin SA, Smith HJ, Engel ME, Fretheim A, Volmink J. Patient adherence to tuberculosis treatment: a systematic review of qualitative research. PLoS Medicine 2007;4(7):e238.

\section{Noyes 2007}

Noyes JP, Popay J. Directly Observed Therapy and TB: how can a systematic review of qualitative research improve services? A qualitative meta-synthesis. Journal of Advanced Nursing 2007;57(3):227-43.

\section{Noyes 2009}

Noyes J, Popay J, Pearson A, Hannes K, Booth A. Chapter 20: Qualitative research and Cochrane reviews. In: Higgins JPT, Green S editor(s). Cochrane Handbook for Systematic Reviews of Interventions Version 5.0.2 [updated September 2009]. The Cochrane Collaboration, 2009.

Oxman 2010

Oxman AD, Bjorndal A, Becerra-Posada F, Gibson M, Block MAG, Haines A, et al.A framework for mandatory impact evaluation to ensure well informed public policy decisions. Lancet 2010;375:427-31.

\section{Rashid 2010}

Rashid C. Benefits and limitations of nurses taking on aspects of the clinical role of doctors in primary care: integrative literature review. Journal of Advanced Nursing 2010;66(8):1658-70.

\section{Rashidian 2008}

Rashidian A, Eccles MP, Russell I. Falling on stony ground? A qualitative study of implementation of clinical guidelines' prescribing recommendations in primary care. Health Policy 2008;85(2):148-61.

\section{Ritchie 1994}

Ritchie J, Spencer L. Qualitative data analysis for applied policy research. In: Bryman A, Burgess R editor(s). Analysing qualitative data. London: Routledge, 1994: 173-94.

\section{Sibbald 2004}

Sibbald B, Shen J, McBride A. Changing the skill-mix of the health care workforce. Journal of Health Services Research \& Policy 2004;9(1):28-38.

\section{Stenner 2010}

Stenner K, Carey N, Courtenay M. Implementing nurse prescribing: a case study in diabetes. Journal of Advanced Nursing 2010;66(3):522-31.

\section{SURE Collaboration 2011}

SURE Collaboration. SURE Guides for Preparing and Using Evidence-Based Policy Briefs: 5. Identifying and addressing barriers to implementing policy options. Version 2.1 [updated November 2011]. The SURE Collaboration 2011; Vol. Available from www.who.int/evidence/sure/ guides.

Thomas 2008

Thomas J, Harden A. Methods for the thematic synthesis of qualitative research in systematic reviews. BMC Medical Research Methodology 2008;8:45.

van Ginneken 2011

van Ginneken N, Tharyan P, Lewin S, Rao GN, Romeo $\mathrm{R}$, Patel V. Non-specialist health worker interventions for 
mental health care in low- and middle- income countries. Cochrane Database of Systematic Reviews 2011, Issue 5.

[DOI: 10.1002/14651858.CD009149]

\section{Volmink 2007}

Volmink J, Garner P. Directly observed therapy for treating tuberculosis. Cochrane Database of Systematic Reviews 2007, Issue 4. [DOI: 10.1002/14651858.CD003343.pub3]

\section{WHO 2004}

World Health Organization. Making pregnancy safer: the critical role of the skilled birth attendant - a joint statement by WHO, ICM and FIGO. Geneva: World Health

Organization, 2004.

\section{WHO 2008}

World Health Organization. Task shifting: rational redistribution of tasks among health workforce teams: global recommendations and guidelines. Geneva: World Health Organization, 2008.

\section{WHO 2012}

World Health Organization. WHO recommendations: Optimizing health worker roles to improve access to key maternal and newborn health interventions through task shifting - OPTIMIZEMNH. Geneva: World Health Organization, 2012.

Wiley-Exley 2007

Wiley-Exley E. Evaluations of community mental health care in low- and middle-income countries: a 10-year review of the literature. Social Science \& Medicine 2007;64(6): $1231-41$.

* Indicates the major publication for the study

\section{ADDITIONAL TABLES}

\section{Table 1. MEDLINE search strategy}

\begin{tabular}{ll}
\hline ID & Search Terms \\
\hline$\# 1$ & triage.tw \\
\hline$\# 2$ & counsel?ing.mp \\
\hline$\# 3$ & counseling.mp \\
\hline$\# 4$ & health education.mp \\
\hline$\# 5$ & health promotion.mp \\
\hline$\# 6$ & $\begin{array}{l}\text { ((professional\$ or nurs\$ or physicians\$ or clinician\$ or GP or doctor? or consultant? or specialist?) adj2 substitut\$). } \\
\text { tw }\end{array}$ \\
\hline$\# 7$ & ((doctor? Or physician? Or consultant? Or specialist?) adj5 nurse? adj5 collaborat\$).tw \\
\hline$\# 8$ & (nurs\$ adj2 run).tw \\
\hline$\# 9$ & (nurs\$ adj2 managed).tw \\
\hline$\# 10$ & (nurs\$ adj2 directed).tw \\
\hline$\# 11$ & (nurs\$ adj2 led).tw \\
\hline$\# 12$ & (professional\$ or nurs\$ or physician\$ or clinicians\$ or GP or doctor? or consultant? or specialist?) adj2 delegat\$).tw \\
\hline 13 & skill mix\$.tw \\
\hline$\#$
\end{tabular}

Barriers and facilitators to the implementation of doctor-nurse substitution strategies in primary care: qualitative evidence synthesis 
Table 1. MEDLINE search strategy (Continued)

\begin{tabular}{|c|c|}
\hline \#14 & (health visitor\$ adj10 (usual care or conventional care)).mp \\
\hline \#15 & (nurs\$ adj10 (usual care or conventional care)).mp \\
\hline \#16 & health visitor\$ adj10 (family or general or doctor? or physician? or practice? or practitioner\$ or GP) \\
\hline$\# 17$ & $\begin{array}{l}\text { nurs } \$ \text { adj10 (family or general or doctor? or physician? or practice? or practitioner \$ or GP or consultant? or specialist? } \\
\text { ) }\end{array}$ \\
\hline \#18 & “nurse run clinic\$”.mp \\
\hline \#19 & team $\$ . m p$ \\
\hline$\# 20$ & role $\$ . m p$ \\
\hline$\# 21$ & cooperat\$.mp. \\
\hline \#22 & exp interprofessional relations/ \\
\hline \#23 & exp "task performance and analyses"/ \\
\hline$\# 24$ & exp professional autonomy/ \\
\hline \#25 & exp *triage/ \\
\hline \#26 & exp * cooperative behavior/ \\
\hline \#27 & exp *delegation, professional/ \\
\hline Substitution & $\begin{array}{l}\# 1 \text { or } \# 2 \text { or } \# 3 \text { or } \# 4 \text { or } \# 5 \text { or } \# 6 \text { or } \# 7 \text { or } \# 8 \text { or } \# 9 \text { or } \# 10 \text { or } \# 11 \text { or } \# 12 \text { or } \# 13 \text { or } \# 14 \text { or } \# 15 \text { or } \# 16 \text { or } \# 17 \text { or } \# 18 \\
\text { or } \# 19 \text { or } \# 20 \text { or } \# 21 \text { or } \# 22 \text { or } \# 23 \text { or } \# 24 \text { or } \# 25 \text { or } \# 26 \text { or } \# 27\end{array}$ \\
\hline \#28 & health visitor $\$ . a b$ \\
\hline $\begin{array}{l}\# 29 \\
\# 30\end{array}$ & $\begin{array}{l}\text { nurs\$.ab } \\
\text { exp nurses/ or nursing staff/ or community health nursing/ or public health nursing }\end{array}$ \\
\hline Nursing & $\# 28$ or \#29 or \#30 \\
\hline \#31 & qualitative.tw. \\
\hline \#32 & themes.tw. \\
\hline Design & $\# 31$ or $\# 32$ \\
\hline
\end{tabular}

Barriers and facilitators to the implementation of doctor-nurse substitution strategies in primary care: qualitative evidence synthesis 


\section{CONTRIBUTIONSOFAUTHORS}

AR, SL, JN and CG devised the study. AR, ES, AKS, CG, JN, SL and CC prepared the protocol. Marit Johansen, AR, ES, AKS, CG and ML developed the search strategy. AR, ES and AKS will actively participate in all stages of the review (conduct search, obtain data, extract data, synthesize data, prepare review, keep the review up to date). CG, JN, SL, CC and ML will participate in reviewing the studies, data synthesis and will contribute to writing up the review.

\section{DECLARATIONS OF INTEREST}

Simon Lewin is an editor for the Cochrane Effective Practice and Organisation of Care Group. Claire Glenton and Simon Lewin are editors for the Cochrane Consumers and Communication Review Group. Jane Noyes is Co-convenor of the Cochrane Qualitative Methods Review Group. The other review authors have no conflicts of interests.

\section{SOURCES OF SUPPORT}

\section{Internal sources}

- No sources of support supplied

\section{External sources}

- Alliance for Health Policy and Systems Research, Implementation Research Platform, Switzerland.

We received funding from the Alliance for Health Policy and Systems Research, Implementation Research Platform: WHO-AHPSR grant 2011/139555-1. 